\title{
MÔ PHỎNG ĐặC TÍNH KHÍ ĐộNG LỰC HỌC MÔ HÌNH AHMED
}

\author{
ĐẶNG TIẾN PHÚC, NGUYỄN THÀNH TÂM, NGUYỄN VĂN SỸ, NGUYỄN XUÂN NGỌC
}

\author{
Khoa Công nghệ Động lục, Trưòng Đại học Công nghiệp thành phố Hồ Chí Minh; \\ dangtienphuc@iuh.edu.vn
}

Tóm tắt. Bài báo này trình bày nghiên cứu đặc tính khí động lực học ô tô thông qua phân tích mô hình xe đơn giản Ahmed. Nghiên cứu được thực hiện trên mô hình Ahmed với các góc nghiêng đuôi xe lần lượt là $\varphi=25^{\circ}$. Tác giả sử dụngphần mềm thương mại $\mathrm{CFD}$ với phương trình RANS kết hợp mô hình rối Realizable $k-\varepsilon$ để mô phỏng đặc tính khí động lực học mô hình Ahmed.Kết quả phân tích thể hiện sự phân bố áp suất, sự phân bố vận tốc quanh mô hình.Kết quả nghiên cứu được thể hiện thông qua các hình ảnh và các giá trị mô phỏng tính toán $C_{\mathrm{d}}, \mathrm{C}_{1}$ tại góc $\varphi=25^{0}$ lần lượt là $\mathrm{C}_{\mathrm{d} 25}=0.291, \mathrm{C}_{125}=0.025$, đồng thời giúp hiểu rõ hơn đặc tính khí động lực học quanh mô hình ô tô đơn giản (mô hình Ahmed).

Từ khóa. Mô phỏng CFD, khí động lực học ô tô, mô hình Ahmed, hệ số cản, RANS.

\section{NUMERICAL SIMULATION OF AERODYNAMIC CHARACTERISTICS OF AHMED MODEL}

\begin{abstract}
This paper studies the aerodynamic characteristics of Ahmed model. Numerical investigations were conducted with the model. The results of the numerical simulations were obtained using Reynoldsaveraged Navier-Stokes (RANS) equations with Realizable $k-\varepsilon$ model. The Time-averaged velocity field, magnitude of the velocity, static pressure magnitude of the flow fields around Ahmed model are presented in this paper. The obtained result of drag coefficient $\left(\mathrm{C}_{\mathrm{d}}\right)=0.291$ and lift coefficient $\left(\mathrm{C}_{\mathrm{l}}\right)=$ 0.025 in $\varphi=25^{\circ}$. The results of this study elucidate the key aerodynamic characteristics around simplified car model (Ahmed model). The results obtained from the study are presented graphically and values of drag coefficient $\left(C_{d}\right)=0.291$, lift coefficient $\left(C_{1}\right)=0.025$ in $\varphi=25^{\circ}$, which helps better understanding aerodynamic characteristics around simplified car model (Ahmed model).
\end{abstract}

Keyword. CFD simulation, Vehicle aerodynamics, Ahmed model, drag coefficient, RANS.

\section{GIỚI THIẸU}

Khí động lực học ô tô đãphát triển trong nhiều thập kỷ qua và đã trở thành một yếu tố quan trọng đối với các dòng xe hơinên rất nhiều các công trình khoa học trong lĩnh vực này được công bố [Hucho, Katz, Halil, Tien Phuc]. Khi ô tô chuyển động trong môi trường không khí sẽ bị các lực và mô ment khí động học tác dụng làm ảnh hưởng đến tính năng chuyển động của ô tô và lượng tiêu hao nhiên liệu. Tổng trọng lượng và hình dạng bên ngoài xe là một trong những yếu tố quan trọng nhằm cải tiến đặc tínhkhí động lực học của xe [Heft, Desai, C. Rajsinh]. Để hiểu được sự tác động khí động lực họclên ô tô, các nhà nghiên cứu đã tiến hành nghiên cứu đặc tính khí động lực học quanh ô tô bằng hai phương pháp mô phỏng và thực nghiệm.

Mô hình ô tô đơn giản với tên gọi Ahmed xuất hiện vào năm 1984 do tác giả Ahmed S.R. đề xuấtđã được nhiều tác giả sử dụng để nghiên cứu đặc tính khí động lực học. Các công trình nghiên cứu đã công bố gần đây, cụ thể tác giả Deepakđã nghiên cứu mô phỏng dòng khí quanh mô hình $2 \mathrm{D}$ Ahmed với góc nghiêng đuôi là $25^{\circ}$ với nhiều mô hình rối khác nhau. Tác giả kết luận rằng các kết quả tính toán mô phỏng vớimô hình rốiRealizable $k-\varepsilon$ cho kết quả tương ứng với kết quả thực nghiệm. Tác giả Wang đã nghiên cứu thực nghiệm mô hình Ahmedvới góc nghiêng đuôi xe lần lượt $25^{\circ}, 35^{\circ}$ trong ống khí động để xác định cấu trúc dòng khí ở sau mô hìnhbằng cách sử dụng phương pháp Particle Image Velocimetry (PIV).Kết quả, tác giả đã minh họa rõ nét và làm sáng tỏ cấu trúc dòng khí tại thời điểm tức thời và tại thời gian trung bình mà trước đó chưa có nghiên cứu nào công bố.Tác giảTunay đã thực hiện nghiên cứu thực nghiệm PIV phân tích sự ảnh hưởng của góc nghiêng đuôi đến đặc tính dòng khí quanh mô hình 
Ahmed.Kết quả, tác giả đã trình bày các hình ảnhcấu trúc dòng khí ở phần đuôi mô hình tại mặt phẳng đối xứng của mô hình Ahmed.Đồng thời tác giả cũng mô tả các điểm tập trung (focus point) và các điểm yên ngựa (saddle point) trong dòng khí ở sau đuôi mô hình. Tác giả Rehan Salahuddin đã sử dụng phần mềm CFD với mô hình rối Realizable $k-\varepsilon$ để phân tích khí động lực học mô hình Ahmed. Kết quả nghiên cứu cho thấy vớigóc nghiêng đuôi $25^{\circ}$, hệ số cản $\mathrm{C}_{\mathrm{d}}=0.32$; góc nghiêng đuôi $35^{\circ}$, hệ số cản $\mathrm{C}_{\mathrm{d}}=0.45$ và các giá trị này khi so sánh với các kết quả thí nghiệm có sai số lần lượt là $1.073 \%$ và $1.703 \%$.

Mặc dù khí động lực học đã được nghiên cứu từ lâu trên thế giới nhưng đây vẫn là lĩnh vực nghiên cứu mà tại Việt Nam chưa được sự quan tâm của các nhà khoa học cũng như các công ty sản xuất, lắp ráp ô tô. Hầu như rất ít hoặc không tìm thấy các nghiên cứu chuyên sâu về khí động lực học ô tô được công bố trên các tạp chí chuyên ngành và các kỷ yếu hội nghị khoa hoc. Vì vậy, tác giả tiến hành nghiên cứu đặc tính khí động lực học ô tô với mô hình đơn giản Ahmed nhằm cung cấp cho người đọc cái nhìn tổng quan và rõ nét hơnsự ảnh hưởng của khí động lực học. Đây cũng chính là một bước quan trọng trong quá trình thiết kế hình dạng ô tô. Để đạt được mục tiêu này, tác giả sử dụng phương trình Reynolds trung bình hóa cho dòng chảy rối(RANS) kết hợp với mô hình rốiRealizable $k-\varepsilon$ dể mô phỏng đặc tính khí động lực học dòng khí.

\section{MÔ HİNH HİNH HỌC AHMED VÀ MIỀN TÍNH TOÁN}

\subsection{Mô hình hình học Ahmed}
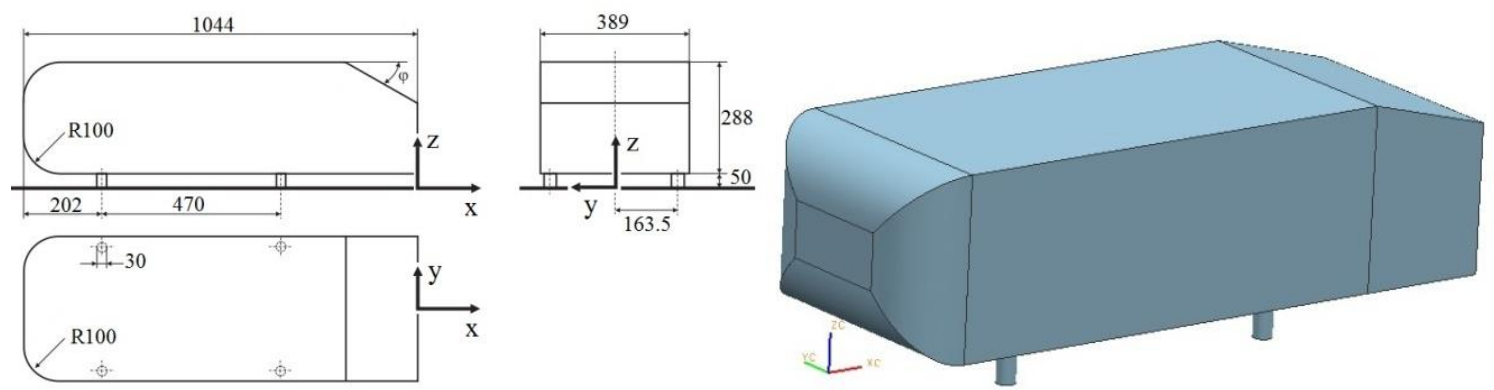

Hình 1. Thông số kỹ thuật mô hình Ahmed 2D (C. Rajsinh) và 3D.

Mô hình Ahmed 2D và 3D được mô tả chi tiết như hình 1 với chiều dài toàn bộ $\mathrm{L}=1044 \mathrm{~mm}$, chiều cao $\mathrm{H}=288 \mathrm{~mm}$, bề rộng toàn bộ $\mathrm{W}=389 \mathrm{~mm}$, bán kính phần đầu mô hình $\mathrm{R}=100 \mathrm{~mm}$, góc nghiêng đuôi $\varphi=25^{0}$, chiều cao chân mô hình $\mathrm{h}=50 \mathrm{~mm}$ trên 3 trục của hệ tọa độ $\mathrm{x}, \mathrm{y}, \mathrm{z}$.
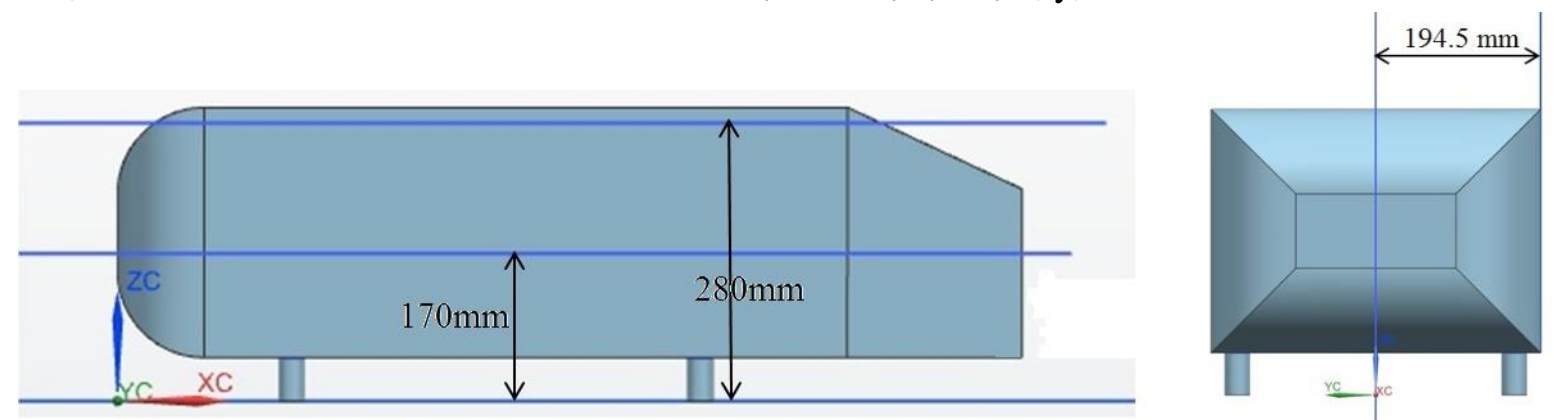

Hình 2. Các mặt phẳng thể hiện sự phân bố áp suất, vận tốc quanh mô hình Ahmed.

Trong quá trình mô phỏng phân tích sẽ thể hiện sự phân bố áp suất, vận tốc quanh mô hình nghiên cứu theo các mặt phẳng mô tả ở hình 2 . Hai mặt phẳng song song với mặt đáy mô hình với các giá trị lần lượt $\mathrm{z}=170 \mathrm{~mm}, \mathrm{z}=280 \mathrm{~mm}$ và mặt phẳng đối xứng của mô hình với giá trị $\mathrm{y}=194.5 \mathrm{~mm}$.

\subsection{Miền tính toán}

Miền tính toánlà vùng không gian bao quanh vật thể được giới hạn trong quá trình mô phỏng.Miền tinh toán phải có kích thước đủ lớn được giới hạn bởi các mặt phẳng để đảm bảo dòng không khí không chịu ảnh hưởng của mô hình nghiên cứu.Tuy nhiên cũng không thể lựa chọn miền tính toán quá lớn dẫn 
đến làm lãng phí tài nguyên máy tính, tăng thời gian mô phỏng tính toán.Vì vậy, kết hợp với các công trình nghiên cứu của các tác giả [Corallo, Tunay, Tien Phuc], tác giả tiến hành xây dựng miền tính toán với các thông số được mô tả ở hình 3.

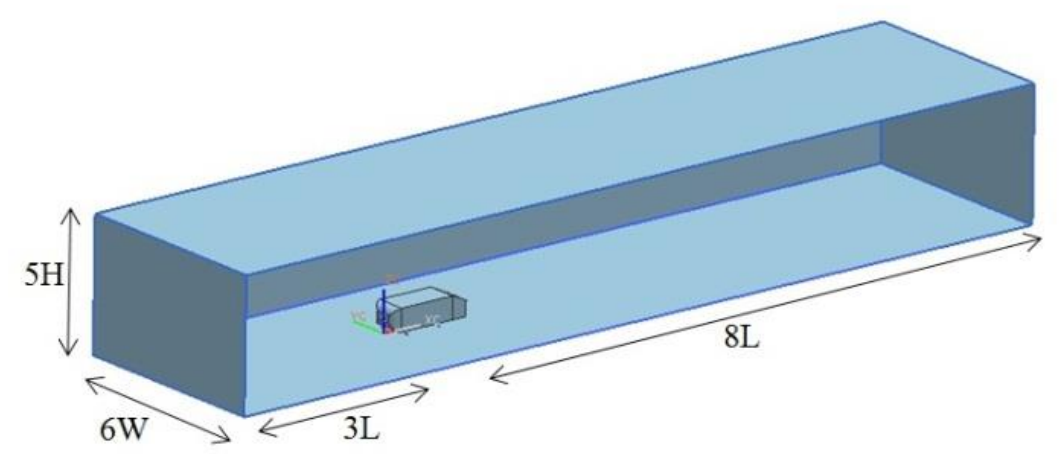

Hình 3. Kích thước của miền tính toán.

\section{PHƯƠNG PHÁP MÔ PHỎNG SỐ}

\subsection{Phương trình điều chỉnh}

Để môtảchuyển động của lưu chất thường sử dụng phương trình Navier-Stokeslà hệ các phương trình bảo toàn khối lượng (phương trìnhliên tục), phương trình bảo toàn động lượng và phương trình bảo toàn năng lượng. Trong nghiên cứu về khí động lực học ô tô đặt giả thiết rằng chất khí không chịu nén, do đó, bài toán khí động lực học chỉ còn lại hai phương trình là phương trình liên tục và phương trình bảo toàn động lượng.

Phương trình liên tục:

$$
\frac{\partial u}{\partial x}+\frac{\partial v}{\partial y}+\frac{\partial \mathrm{w}}{\partial z}=0
$$

Phương trình bảo toàn động lượng:

$$
\partial_{t} u_{i}+u_{j} \partial_{j} u_{i}=-\frac{1}{\rho} \partial_{i} p+v \partial_{j} \partial_{j} u_{i}
$$

Trong bài nghiên cứu này, tác giả sử dụng phương trình Reynolds Navier - Stokes trung bình hóa (RANS) để giải bài toán khí động học:

$$
\begin{aligned}
& \partial_{i} \bar{u}_{i}=0 \\
& \partial_{t} \bar{u}_{i}+\bar{u}_{j} \partial_{j} \bar{u}_{i}=-\frac{1}{\rho} \partial_{i} \bar{p}+\frac{1}{\rho} \partial_{j}\left(\tau_{\mathrm{ij}}-\rho \overline{u_{i}^{\prime} u_{i}^{\prime}}\right)
\end{aligned}
$$

Ngoài ra, để giải phương trình RANS tác giả kết hợp với mô hình rối Realizable $k-\varepsilon$.

\subsection{Chia lưới và điều kiện biên}

Chia lưới chính là rời rạc hóa vùng không gian mô phỏng thành các phần tử để thực hiện tính toán gần đúng bằng phương pháp số. Lưới có hai dạng đó là lưới có cấu trúc và lưới không có cấu trúc và mỗi loại đều có những điểm mạnh riêng. Trong bài nghiên cứu này sẽ sử dụng lưới tứ diện không có cấu trúc do mô hình mô phỏng phức tạp. Miền tính toán được chia lại với kích thước của phần tử lưới ở vùng biên và gần mô hình phân tích phải đủ nhỏ để đảm bảo độ chính xác của kết quả khi mô phỏng tính toán (Katarzyna, Halil), trong khi các vùng xa vùng biên và mô hình phân tích kích thước của phần tử lưới lớn hơn nhẳm tiết kiệm tài nguyên của máy tính cũng như thời gian tính toán. Mô hình Ahmed đã chia lưới hoàn chỉnh được mô tả ở hình 4. 


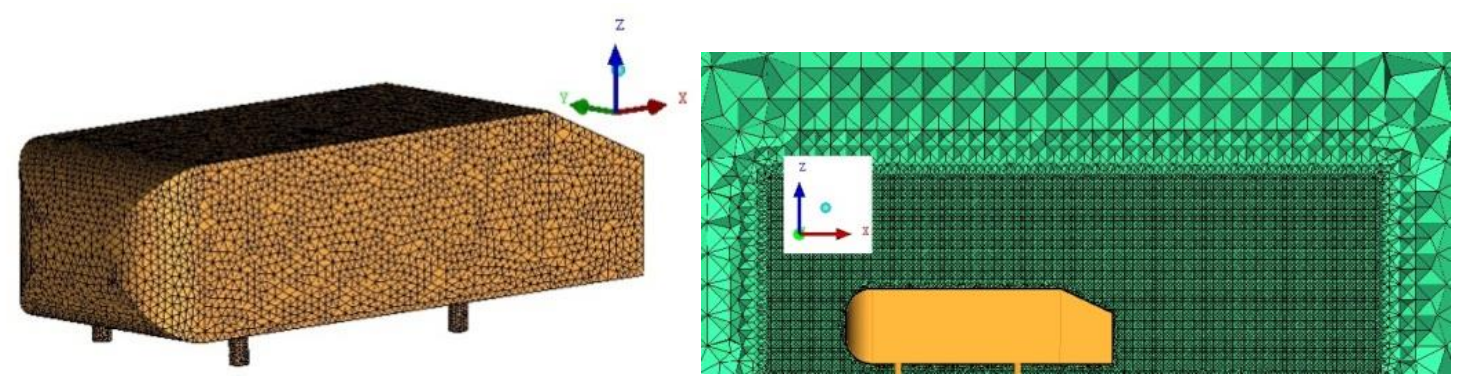

Hình 4. Mô hình Ahmed đã chia lưới với dạng tứ diện không có cấu trúc

Để xác định số lượng phần trong miền tính toán bao nhiêu là phù hợp, tác giả tiến hành thực hiện kiểm tra lưới dựa vào kêt quả tính toán $\mathrm{C}_{\mathrm{d}}$ rồi so sánh với kết quả thực nghiệm $\mathrm{C}_{\mathrm{d}}=0.298$ của tác giả Ahmed S.R. trong công trình nghiên cứu trước và thời gian tính toán.Sau khi chia lưới xong, tiến hành đặt các điều kiện biên để mô phỏng tính toán kết quả. Vận tốc dòng khí được thiết lập là $30 \mathrm{~m} / \mathrm{s}$, số Reynolds được xác định dựa vào chiều dài của mô hình Ahmed là 2.144x10 giải bài toán liên kết giữa thành phần vận tốc và áp suất trong phương trình RANS.Kết quả, số lượng các phần tử trong miền tính toán thuộc trường hợp 4 được lựa chọn là khoảng $6.758 \times 10^{5}$ phần tử vì giá trị $\mathrm{C}_{\mathrm{d}}$ được tính toán xấp xỉ với giá trị $\mathrm{C}_{\mathrm{d}}$ tham chiếu và thời gian tính toán nhanh hơn.

Bảng 1. Bảng lựa chọn số phần tử lưới ứng với giá trị tính toán hệ số cản $\mathrm{C}_{\mathrm{d}}$.

\begin{tabular}{|l|c|c|c|c|c|}
\hline Dữ liệu & Trường hợp 1 & Trường hợp 2 & Trường hợp 3 & Trường hợp 4 & Trường hợp 5 \\
\hline Số phần tử & $3.731 \times 10^{5}$ & $4.678 \times 10^{5}$ & $5.734 \times 10^{5}$ & $6.758 \times 10^{5}$ & $7.792 \times 10^{5}$ \\
\hline $\mathrm{C}_{\mathrm{d}}$ & 0.265 & 0.271 & 0.280 & 0.291 & 0.290 \\
\hline Thời gian(h) & 3 & 4 & 4,5 & 5 & 8 \\
\hline
\end{tabular}

\section{KẾT QUẢ}

\subsection{Phân bố trường vận tốc quanh mô hình Ahmed theo thời gian trung bình}

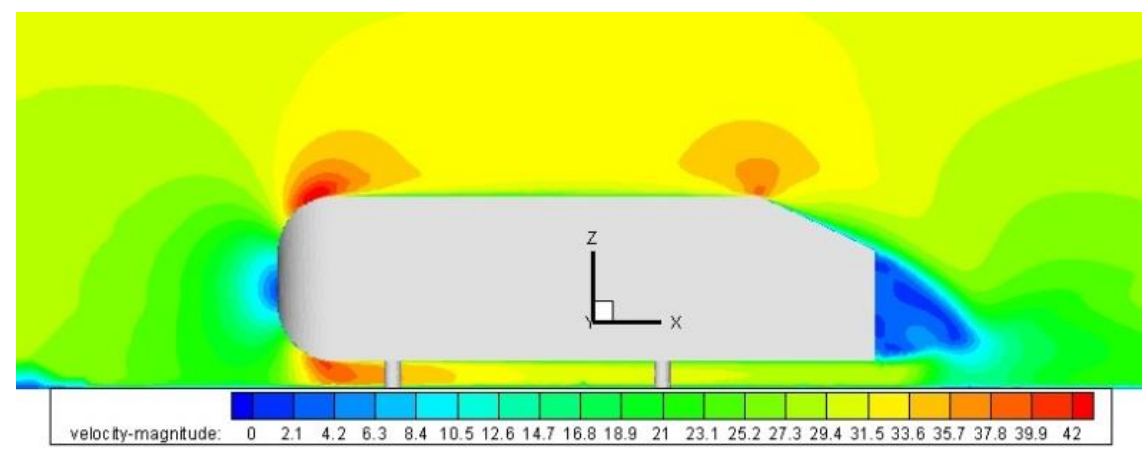

Hình 5. Phân bố vận tốc tại mặt phẳng dọc đối xứngcủa mô hình Ahmed.

Hình 5 thể hiện trường vận tốc tại mặt phẳng dọc đối xứng của mô hình Ahmed với bước lặp thời gian là $2 \times 10^{5}$.Thang màu sắc từ xanh da trời đến màu đỏ thể hiện độ lớn vận tốc tại những vùng khác nhau quanh mô hình. Tại vùng đầu mô hình luôn luôn có vận tốc nhỏ hơn ở những vùng khác trên mô hình do dòng khí khi di chuyển đến vùng này sẽ xuất hiện một điểm đình trệ (stagnation point). Ngay tại điểm đình trệ vận tốc dòng khí bằng không và dòng khí sẽ chia làm hai phần, một phần đi dọc theo phía trên thân mô hình và một phần đi dọc theo phía dưới thân mô hình. Bên cạnh đó, biên dạng mô hình thay đổi (các góc bo tròn, góc nghiêng) dẫn đến sự phân bố vận tốc tại những vùng đó cũng thay đổi.Vùng sau đuôi mô hình Ahmed hình thành xoáy thấp áp ảnh hưởng đến lực cản khí động của mô hình, vậy để giảm lực cản khí động cần phải loại bỏ hoặc giảm kích thước vùng xoáy. 


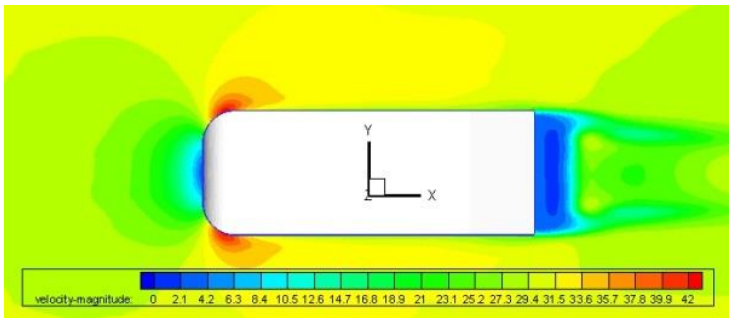

$\mathrm{z}=170 \mathrm{~mm}$

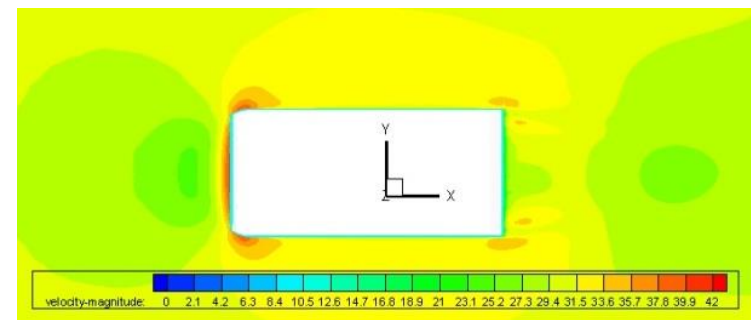

$\mathrm{z}=280 \mathrm{~mm}$

Hình 6. Phân bố vận tốc tại mặt phẳng tại mặt phẳng cắt ngang của mô hình Ahmed.

Hình 6 thể hiện hiện trường vận tốc tại mặt phẳng cắt ngang đi qua điểm giữa của mô hình Ahmed và mặt phẳng này cách phần thấp nhất của mô hình lần lượt là $\mathrm{z}=170 \mathrm{~mm}, \mathrm{z}=280 \mathrm{~mm}$. Tại vùng phía sau mô hình, góc nghiêng đuôi làm ảnh hưởng đến sự phân bố vận tốc.

\subsection{Phân bố áp suấtquanh mô hình Ahmed}
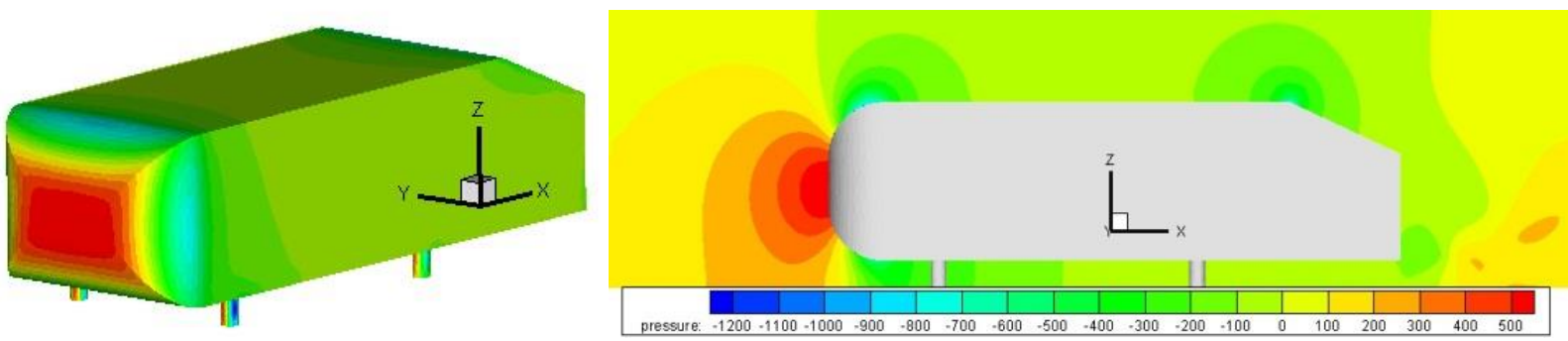

Hình 7. Phân bố áp suất trên mô hình Ahmed và tại mặt phẳng đối xứngdọc.

Hình 7thể hiện sựphân bố áp suất trên mô hình Ahmed và tại mặt phẳng đối xứngdọc quanh mô hình.Thang màu sắc từ xanh da trời đến màu đỏ thể hiện độ lớn áp suất tại những vùng khác nhau quanh mô hình.Tại phía trước mô hình luôn có áp suất lớn hơn ở những vùng khác trên mô hình do sự tách rời của dòng khí, chính sự chênh áp này là yếu tố cơ bản tạo nên lực cản khí động khi ô tô di chuyển về phía trước. Sự chênh áp càng lớn thì lực cản khí động càng tăng và để làm giảm lực cản khí động thì cần tăng áp suất phía sau mô hình.

Quan sát hình 4 và hình 5 ta thấy tại những vị trí có vận tốc thay đổi thì áp suất cũng thay đổi hoặc ngược lại. Cụ thể, tại vùng phía trước mồ hình vận tốc nhỏ thì áp suất tại đó cao. Như vậy, sự phân bố vận tốc và áp suất quanh mô hình luôn tuân theo định luật Bernoulli.

\subsection{Lực khí động lực học}

Theo kết quả tính toán mô phỏng mô hình Ahmed trong ống khí động, kết quả tính toán các giá trị $\mathrm{C}_{\mathrm{d}}, \mathrm{C}_{\mathrm{l}}$ được trình bày ở bảng 2. Giá trị $\mathrm{C}_{\mathrm{d}}$ được tính toán từ kết quả mô phỏng nhỏ hơn giá trị $\mathrm{C}_{\mathrm{d}}$ tham khảo từ kết quả thực nghiệm với sai số $2.405 \%$.Từ các giá trị $\mathrm{C}_{\mathrm{d}}, \mathrm{C}_{\mathrm{l}}$ tiến hành tính được các lực cản thông qua công thức:

$$
\begin{array}{lll}
\text { Lực cản không khí: } & F_{d}=\frac{1}{2} C_{d} A \rho U_{\infty}{ }^{2} \\
\text { Lực nâng: } & F_{l}=\frac{1}{2} C_{l} A \rho U_{\infty}{ }^{2}
\end{array}
$$

Bảng 2. Kết quả tính toán hệ số nâng, hệ số cản của mô hình.

\begin{tabular}{|l|c|c|}
\hline Các hệ số & $\mathrm{C}_{\mathrm{d}}$ & $\mathrm{C}_{1}$ \\
\hline Kết quả mô phỏng & 0.291 & 0.025 \\
\hline
\end{tabular}




\section{KẾT LUẬN}

Bài báo đã trình bày kết quả tính toán mô phỏng đặc tính khí động lực học cho mô hình ô tô đơn giản Ahmed, sử dụng phương trình RANS kết hợp với mô hình rối Realizable $k-\varepsilon$ đối với mô phỏng CFD.Sai số giữa kết quả tính toán mô phỏng giá trị $\mathrm{C}_{\mathrm{d}}$ với giá trị thực nghiệm là $2.34 \%$. Bên cạnh đó, bài báo này cũng đã minh họa rõ bằng hình ảnh sự phân bố áp suất, vận tốc quanh mô hình trên các mặt phẳng khác nhau. Kết quả nghiên cứu là tiền đề để thực hiện tính toán mô phỏng trên mô hình ô tô thực tế, thông qua mô phỏng số CFD sẽ giải quyết được vấn đề thí nghiệm khí động lực học ô tô khi hiện nay phòng thí nghiệm khí động lực học ô tô ở nước ta còn rất hạn chể.

\section{Các ký hiệu}

- u, v,w: Vận tốc của phần tử lưu chất theo 3 trục tọa độ $\mathrm{x}, \mathrm{y}, \mathrm{z}$.

- $\partial_{t} u_{i} \quad$ : Đạo hàm riêng theo thời gian của $\mathrm{u}$.

- $\rho:$ Khối lượng riêng của không khí $\left(\mathrm{kg} / \mathrm{m}^{3}\right)$.

- p: Áp suất $\left(\mathrm{N} / \mathrm{m}^{2}\right)$.

- $\quad v$ : Độ nhớt động học của không khí $\left(\mathrm{kg} \cdot \mathrm{m} / \mathrm{s}^{-1}\right)$.

- $\mathrm{C}_{\mathrm{d}}$ : Hệ số cản.

- $\mathrm{C}_{1}$ : Hệ số nâng.

- $\rho \overline{u_{i}^{\prime} u_{i}^{\prime}}$ : Úng suất Reynolds.

$-\tau_{\mathrm{ij}} \quad$ : Ten sơ ứng suất

- A : Diện tích cản chính diện $\left(\mathrm{m}^{2}\right)$

- $U_{\infty} \quad$ : Vận tốc chuyển động $(\mathrm{m} / \mathrm{s})$

\section{TÀI LIỆU THAM KHẢO}

1. Ahmed S, Ramm G, Faitin G, Some salient features of the time-averaged ground vehicle wake. SAE Technical Paper 840300, 1984, doi:10.4271/840300.

2. C. Rajsinh B. and T. K. Raj R., Numerical investigation of external flow around the ahmed reference body using computational fluid dynamics, Research Journal of Recent Sciences, 2012, 1(9): 1-5.

3. D. Tienphuc, Gu zhengqi, Chenzhen, Numerical Simulation of the Flow Field around Generic Formula One, Journal of Applied Fluid Mechanics, 2016, 9(1):443-450.

4. Deepak K. Kalyan, A.R. Paul, Computational Study of Flow around a Simplified 2D Ahmed Body, International Journal of Engineering Science and Innovative Technology (IJESIT), 2013, 2(3): 148-153.

5. Halil, S.H., Rami, S.E., Murat, A. and Ibrahim, D, Effects of rear spoilers on ground vehicle aerodynamic drag", International Journal of Numerical Methods for Heat \& Fluid Flow,2014, 24(3): 627-642.

6. Halil, S.H., Rami, S.E., Murat, A. and Ibrahim, D., Effects of rear spoilers on ground vehicle aerodynamic drag, International Journal of Numerical Methods for Heat \& Fluid Flow, 24(3): 627-642.

7. Heft, A., Indinger, T., and Adams, N., Introduction of a New Realistic Generic Car Model for Aerodynamic Investigations, SAE Technical Paper 2012, doi:10.4271/2012-01-0168.

8. Hucho, W.H., Aerodynamics of Road Vehicles, Butterworth and Co. Publishing, Boston, MA, 1990.

9. Katarzyna, S., Gabriel, W. and Derek, B.I., CFD modelling of air and oxy-coal combustion, International Journal of Numerical Methods for Heat \& Fluid Flow, 2014, 24( 4):825-844.

10. Katz, J., Race Car Aerodynamics: Designing for Speed, Bentley Publishers, Philadelphia, PA, 1995. 
11. M. Desai, S. A. Channiwala and H. J. Nagarsheth, A Comparative assessment of two experimental methods for aerodynamics performance evaluation of car,Journal of scientific and industrial Research, 2008, 67: 518-522.

12. M. Corallo, J. Sheridan, M.C. Thompson, Effect of aspect ratio on the near-wake flow structureof an Ahmed body, Journal of Wind Engineering and Industrial Aerodynamics, 2015, 147:95-103.

13. Rehan Salahuddin Khan, Sudhakar Umale, CFD Aerodynamic Analysis of Ahmed Body, International Journal of Engineering Trends and Technology (IJETT), 2014, 18 ( 7): 301-308.

14. Tien Phuc Dang, Zhengqi Gu, Zhen Chen, Numerical simulation of flow field around the race car in case, International Journal of Numerical Methods for Heat \& Fluid Flow, 2015, 25(8): 896- 1911.

15. Tural Tunay, Bulent Yaniktepe, Besir Sahin, Computational and experimental investigations of the vortical flow structures in the near wake region downstream of the Ahmed vehicle model, Journal of Wind Engineering and Industrial Aerodynamics, 2016, 159:48-64.

16. Tural Tunay, Besir Sahin, Veli Ozbolat, Effects of rear slant angles on the flow characteristics of Ahmed body, Experimental Thermal and Fluid Science, 2014, 57: 165-176.

17. Wang, X.W., Zhou, Y., Pin, Y.F., Chan, T.L., Turbulent near wake of an Ahmed vehicle model. Exp. Fluids, 2013, 54:1490, DOI: 10.1007/s00348-013-1490-x.

Ngày nhận bài: 01/04/2017

Ngày chấp nhận đăng: 20/05/2017 